\title{
The Steady States of a Stochastic Decentralized Growth Model
}

\author{
JOHN LAITNER \\ Department of Economics, University of Michigan, \\ Ann Arbor, Michigan 48109
}

Received April 20, 1979; March 24, 1980

The purpose of this paper is to prove the existence of a steady state for a simple decentralized growth model which is subject to random shocks. The model has the following elements. The population is composed of an endless sequence of overlapping cohorts of two-period households; each cohort is the same size; and, there is an aggregate production function and one output good. Because the labor supply is fixed, the production function relates output in each period $t$ to the total saving of all young families in period $t-1$ and to the current realization $z_{t}$ of a random variable $\tilde{z}$. The random variable has an exogenous, unchanging, and known distribution. Futures markets are imperfect: young families can save at time $t$ only by investing in physical capital, which will pay a return at time $t+1$. The return depends on the size of the time- $(t+1)$ capital stock, $k_{t}$, and on $z_{t+1}$. Let $y_{t}=y\left(k_{t-1}, z_{t}\right)$ be the vector of incomes of young families at time $t$. Then such families use a function $g\left(y_{t}\right)=k_{t}$ to predict $k_{t}$ (note that $g$ is nonstochastic). The families adjust their saving to maximize their expected utility, where the latter is computed using $g$ and the distribution function for $\tilde{z}$. We call resulting aggregate savings by young families $\gamma\left(y_{t}, g\right)$.

We use a fixed-point argument to prove the existence of sets $K$ and $Y$ and an expectation function $g$ such that if all realizations of $\tilde{z}$ fall within $Z$, (i) $y(K \times Z) \subset Y$, (ii) $g(Y) \subset K$, and (iii) $g(y)=\gamma(y, g)$ all $y \in Y$. The resulting $g$ determines (in a very simple context) what Grandmont [7] would call "rational' expectations" and what Radner $[17,18]$ would call an "equilibrium of plans and expectations." We use this $g$ to generate a transition function $v$ for $k_{t}$. We then show $v$ has a finite invariant set $K^{*}$ (which includes all feasible states except one trivial one); there are a finite number of ergodic sets $E_{1}, \ldots, E_{N} \subset K^{*}$ for $v$; and there is a unique stationary distribution function $D_{i}$ associated with each $E_{i}$. Each pair $\left(D_{i}, E_{i}\right)$ constitutes a "steady state" (corresponding to Green and Majumdar's [9] concept of an "equilibrium distribution" and to Grandmont's [7] "long-run equilibrium"): Once the evolution of the economy carries it into $E_{i}$ some 
$i \in\{1, \ldots, N\}$, it will remain in $E_{i}$ in each subsequent period with probability 1. If the economy has entered $E_{i}, \int_{A} D_{i}(d x)$ gives the long-term frequency of appearance for states in any Borel subset $A \subset E_{i}$. States in $K^{*}-\left(\bigcup_{i=1}^{N} E_{i}\right)$ are transitory, leading to entrance into some $E_{i}$ after a finite number of periods with probability 1 .

The novelty of our analysis lies in our ability to characterize $v$ solely on the basis of restrictions on family preference orderings and the aggregate production function. ${ }^{1}$ Our work can be viewed as a complement to Mirman's $[14,15]$ competitive analysis and to Brock and Mirman's [4] optimal growth model. Mirman's papers postulate the existence of an aggregative saving function with various properties; Brock and Mirman derive such a function from a model with a single, centrally controlled maximization process. In this paper, utility maximization on the part of individual families leads to an overall saving function. ${ }^{2}$

The organization of this paper is as follows. Section I presents the assumptions of our model. Section II proves the existence of an equilibrium transition function $v$ (in other words, the existence of a transition function consistent with rational expectations on the part of all families and zero excess demand in each period). Our proof is somewhat involved because we need $v$ to have strong enough properties to establish the existence of a steady state in Section III. A mathematical appendix at the end of the paper supplies proofs for all lemmas and propositions.

\section{THE Model}

We first present our basic assumptions about preferences and production. Then we list several special restrictions needed for particular steps in this paper. At the end of this section we prove two lemmas which we will use to construct an invariant set $\left[k_{L}, k_{U}\right] \subset(0, \infty)$ for values of the aggregate capital stock.

We assume that at each time $t$ the population of the economy consists of an equal number of young and old families. The number remains fixed over time. Although the population is finite, we assume that it is so large that with no discernible influence on its own well-being each individual family can behave as though its saving decision will not affect the next-period wage rate and rate of return on capital. The families in each cohort are indexed with numbers $j \in\{1, \ldots, J\}$. Each family $j$ has a utility function $u\left(c_{1}, c_{2}, j\right)$ with $c_{1}$

\footnotetext{
'See, in particular, Grandmont's comments in Sections 5.1-5.3 of [7] and Radner's comments in Section 4.1 of [18].

${ }^{2}$ Inaba [11] uses a model somewhat in the same vein. However, he does not allow production and he does place direct restrictions on aggregative demand functions.
} 
consumption in the first period of life and $c_{2}$ consumption in the second. The family's endowment consists of a supply of "effective" labor units in each period of life, $\left(l_{1}(j), l_{2}(j)\right)$. We assume that the number of families of each type $\left(u(\cdot) ; l_{1}(\cdot), l_{2}(\cdot)\right)$ remains the same generation after generation. We also assume $l_{1}(j)>0$ and $l_{2}(j) \geqslant 0$ each $j$.

We want $u: R^{2} \times\{1, \ldots, J\} \rightarrow R$ to have the following properties in every case:

(A1) For each $\left(c_{1}, c_{2}\right) \in(0, \infty) \times(0, \infty), u$ is twice continuously differentiable in $c_{1}$ and $c_{2}, \lim _{c \downarrow 0} u_{1}\left(c, c_{2}, j\right)=\lim _{c \downarrow 0} u_{2}\left(c_{1}, c, j\right)=\infty$, $u_{1}\left(c_{1}, c_{2}, j\right)>0, u_{2}\left(c_{1}, c_{2}, j\right)>0, u_{12}\left(c_{1}, c_{2}, j\right) \geqslant 0$, and $u$ is concave in $c_{1}$ and $c_{2}$.

Any utility function $u: R^{2} \rightarrow R$ of the Bergson class, for example, would satisfy (A 1$)$.

Let $k_{t}$ be the total saving of all young families at time $t$. Since the total supply of "effective" labor units is unvarying, production next period, $q_{t+1}$, can be written

$$
q_{t+1}=f\left(k_{t}, z_{t+1}\right)
$$

where $z_{t+1}$ is a random realization of $\tilde{z}$. This formulation is consistent with the spirit of Mirman's [14,15] and Brock and Mirman's [4] familiar analyses. We assume that $z_{t}$ all $t$ represent random samplings from $\tilde{z}$, that $z_{t} \in R$ all $t$, and that

(A2) There exists an interval $Z=\left[z_{L}, z_{U}\right] \subset(-\infty, \infty)$ with $\int_{z} P(d z)=1$, where $P$ is the distribution function of $z$.

Since there is only one good available in each period, we can think of its current price as always being 1 . Then assuming constant returns to scale and competitive conditions, if $w_{t}$ is the wage rate per "effective" unit of labor at time $t$ and $r_{t}$ is one plus the interest rate (net of depreciation),

$$
\begin{aligned}
& w_{t+1}=w\left(k_{t}, z_{t+1}\right)=f\left(k_{t}, z_{t+1}\right)-f_{1}\left(k_{t}, z_{t+1}\right) \cdot k_{t}, \\
& r_{t+1}=r\left(k_{t}, z_{t+1}\right)=f_{1}\left(k_{t}, z_{t+1}\right) .
\end{aligned}
$$

We also assume

(A3) $f$ is twice continuously differentiable. For each $k>0$ and $z \in Z$ we have $f(k, z)>0, r(k, z)>0, w(k, z)>0, w_{1}(k, z)>0$, and $r_{1}(k, z)<0$. We also assume $\lim _{k \rightarrow \infty} r(k, z)<1$ and $\lim _{k \rightarrow 0} r(k, z)=\infty$ any $z \in Z$. 
We need three specialized assumptions. The first will enable us to prove the existence of an ergodic set for $k$ other than $\{0\} .{ }^{3}$ If $l$ is the total supply of "effective" labor units per period, let

$$
\sigma(k, z)=r(k, z) \cdot k /[w(k, z) \cdot l] .
$$

Let "lim" stand for " $\lim _{n \rightarrow \infty}$ " "Then

(A4) For at least one $j$, given any sequence $\left\{\left(\alpha(n), \alpha^{*}(n)\right)\right\}$ with $\lim \left(\alpha(n), \alpha^{*}(n)\right)=(0,0)$, if $\lim u_{1}\left(\alpha(n), \alpha^{*}(n), j\right) / u_{2}\left(\alpha(n), \alpha^{*}(n), j\right)=\infty$, we must have $\lim \alpha^{*}(n) / \alpha(n)=\infty$. Also, $\lim _{k \mid 0} \sigma(k, z) \in[0, \infty)$ any $z \in Z$, and $\lim _{k \downarrow 0} w(k, z) / w\left(k, z^{\prime}\right) \in(0, \infty)$ any $z, z^{\prime} \in Z$.

The first part of this assumtion requires that if we graph the isoquant map for $u$, moving through the positive orthant toward the origin, the marginal rate of substitution must not diverge to $\infty$ unless the path we follow converges to the $c_{2}(j)$ axis. Homothetic utility functions, for instance, satisfy this restriction (given (A1)). CES production functions with elasticity of substitution $\geqslant 1$ will satisfy the second part of the assumption.

In order to prove the existence of an equilibrium transition function with several essential properties in Section II, we need

$$
d[r(k, z) \cdot k] / d k>0 \quad \text { all } k \in K, z \in Z,
$$

where $K$ is a bounded interval to be defined shortly. This assumption ensures that an additional dollar's worth of saving at time $t$ enlarges property income at time $t+1$ regardless of the value $z_{t+1}$ takes (provided $k_{t} \in K$ ). A production function of the form $f(k, z)=z \cdot f^{*}(k)$ with $f^{*}(\cdot)$ Cobb-Douglas or CES with elasticity of substitution $>1$ will satisfy (A5).

Assumptions (A1) to (A5) are sufficient to establish the existence of at least one invariant measure for the economy's equilibrium transition function $v$. The next assumption insures that the number of ergodic sets for each such $v$ is finite and that none contain two or more cyclic subsets.

(A6) $z^{\prime}>z$ implies $f\left(k, z^{\prime}\right)>f(k, z), r\left(k, z^{\prime}\right)>r(k, z)$, and $w\left(k, z^{\prime}\right)>$ $w(k, z)$ all $k>0 ; z, z^{\prime} \in Z$. $\tilde{z}$ has a continuous density function $p$, and $p(z)>0$ all $z \in Z$.

Any production function of the form $f(k, z)=z \cdot f^{*}(k)$ with $f^{*}(\cdot)$ having conventional "neoclassical properties" will satisfy (A6).

\footnotetext{
${ }^{3}$ If $f(0, z)=0$ all $z \in Z$, then $\{0\}$ is one ergodic set for our cconomy. Wc will not devotc any attention to this particular ergodic set, however.
} 
At the start of period $t$ the state variable $k_{t-1}$ summarizes the past history of the economy relevant for determining $w_{t} \cdot l_{1}(j)$ each $j$. Let $y: R^{2} \rightarrow R^{J}$ be

$$
y(k, z)=w(k, z) \cdot\left(l_{1}(1), \ldots, l_{1}(J)\right) .
$$

Provided young families at time $t$ know $z_{t}$ as they decide on $c_{1}(j)$ all $j, k_{t}$ depends on $y_{t}=y\left(k_{t-1}, z_{t}\right)$ and expectations. We assume that all such families know $y_{t}$ and use $g: R^{J} \rightarrow R$ with

$$
g\left(y_{t}\right)=k_{t}
$$

to predict $k_{t}{ }^{4}$ Then assuming that no family's net worth can ever become negative and that families attempt to maximize their expected utility, each family at time $t$ chooses $c_{1}(j)$ to solve

$$
\operatorname{Max}_{\left\{c_{1}(j)\right\}} \int_{z} u\left(c_{1}(j), c_{2}(j), j\right) P(d z)
$$

subject to:

$$
\begin{aligned}
c_{1}(j)+c_{2}(j) / r_{t+1} & =l_{1}(j) \cdot w_{t}+l_{2}(j) \cdot w_{t+1} / r_{t+1}, \\
r_{t+1} & =f_{1}\left(g\left(y_{t}\right), z\right), \\
w_{t+1} & =f\left(g\left(y_{t}\right), z\right)-f_{1}\left(g\left(y_{t}\right), z\right) \cdot g\left(y_{t}\right), \\
c_{1}(j) & \leqslant l_{1}(j) \cdot w_{t} .
\end{aligned}
$$

Suppose that

$$
\gamma\left(y_{t}, g\right)=k_{t}
$$

gives the actual value of $k_{t}$ if all families base their expectations on $g$. Then the next section shows that for the sets $K \subset R$ and $Y \subset R^{J}$ defined below there is at least one $g: R^{J} \rightarrow R$ such that (i) $g(Y) \subset K$; (ii) $y(K \times Z) \subset Y$; and (iii) $g(y)=\gamma(y, g)$ all $y \in Y$. Thus for capital-to-labor ratios in $K$, the income vector for young families must lie in $Y$. For all income vectors in $Y$, however, anticipated next-period capital-to-labor ratios will again be in $K$ and the anticipations will be borne out exactly.

To construct the sets $Y$ and $K$ we need two lemmas.

Lemma 1. There exists $k_{U} \in(0, \infty)$ such that $0<k \leqslant k_{U}$ implies $\gamma(y(k, z), g) \leqslant k_{U}$ any $g: R^{J} \rightarrow R$ and all $z \in Z$.

${ }^{4}$ Notice that $k_{t}$ is not a stochastic variable here. Notice also that there is no benefit to one family of using observations of $y_{t-s}$ any $s \geqslant 1$ in predicting $k_{t}$ (unless it knows other families do). 
LEMMA 2. For any $k_{L}<k_{U}$ let $K=K\left(k_{L}\right)=\left\{k_{L}, k_{U}\right\}$. Let $Y=$ $\prod_{j=1}^{J}\left[y_{L}(j), y_{U}(j)\right]$, where $y_{L}(j)=w\left(k_{L}, z_{L}\right) \cdot l_{1}(j)$ and $y_{U}(j)=w\left(k_{U}, z_{U}\right)$. $l_{1}(j)$ all $j .{ }^{5}$ Suppose $y_{L}=\left(y_{L}(1), \ldots, y_{L}(J)\right)$ and $g: Y \rightarrow K$. Then there exists $k_{L} \in\left(0, k_{U}\right)$ such that $g\left(y_{L}\right)=k_{L}$ implies $\gamma\left(y_{L}, g\right)>k_{L}$.

The same $k_{L} \in\left(0, k_{U}\right)$ works for any $g: Y \rightarrow K$. We fix $k_{L}, k_{U}, K$ and $Y$ as they are defined in the lemmas.

\section{EQuiLIBRIUM}

The operator $\gamma$ (see line (6)) associates with each function $g: Y \rightarrow K$ a second function

$$
g^{*}=\Gamma(g)
$$

with $g^{*}(y)=\gamma(y, g)$ all $y \in Y$. Each fixed point for $\Gamma$ defines an equilibrium (with rational expectations) for our model: if all families' expectations are given by $g$, the actual evolution of the economy is governed by $g^{*}=\Gamma(g)$. It is not difficult to prove that $\Gamma$ must have at least one such fixed point. ${ }^{6}$ In order to complete the analysis of steady states in Section III, however, we seek a fixed point $g=\Gamma(g)$ which is continuous and strictly monotone.

Our argument, therefore, requires several steps. Letting $Y$ and $K$ be as in Section $I$, and letting $\Delta=\left(\Delta_{1}, \ldots, \Delta_{J}\right)$, define

$$
\begin{aligned}
G(\varepsilon)= & \{g: Y \rightarrow K \mid g \text { is continuous, } 0 \leqslant g(y+\Delta)-g(y) \leqslant \delta \cdot \varepsilon \\
& \text { all } \left.y, y+\Delta \in Y \text { with } \Delta_{j}=\delta \text { some } j \text { and } \Delta_{i}=0 \text { all } i \neq j\right\} .
\end{aligned}
$$

Then if $\|\cdot\|$ is the uniform norm on $Y,\|g-\bar{g}\|=\sup _{y \in Y}|g(y)-\bar{g}(y)|$,

Lemma 3. For any $\varepsilon>0, G(\varepsilon)$ is compact in the topology induced by $\|\cdot\|$.

Although $\Gamma$ does not necessarily take $G(\varepsilon)$ into itself, we construct a continuous operator, $\Psi$, that does. For each $\varepsilon>0$ the new function must have a fixed point on $G(\varepsilon)$. We show that with the correct choice of $\varepsilon$, however, all fixed points of $\Psi$ on $G(\varepsilon)$ must also be fixed points of $\Gamma$.

${ }^{5}$ Although we are assuming that (A6) holds as we define $y_{L}(j)$ and $y_{L}(j)$, we could avoid the need to do that by defining $y_{L}(j)=w^{*}\left(k_{L}\right) \cdot l_{1}(j)$, where $w^{*}\left(k_{L}\right)=\operatorname{Min}_{z \in Z} w\left(k_{L}, z\right)$, etc. The same applies later in the paper (until Section III) and in the Appendix.

${ }^{6}$ To see this, let $H=\{g: Y \rightarrow K\}$. Then $H$ is compact in the topology of pointwise convergence and $\Gamma$ is continuous on $H$ with that topology. If $g \in H$ and $g^{*}=\Gamma(g)$. $g^{*}(y)<k_{U}$ all $y \in Y$ by Lemma 1. If $g(y)=k_{L}$, the proof of Lemma 2 can be modified to show $g^{*}(y)>k_{L}$ regardless of the choice of $y \in Y$. Thus, $\Gamma(\partial H) \subset H$. So, $\Gamma$ has a fixed point on $H$. 
First we establish three properties of any $g^{*}=\Gamma(g)$ with $g \in G(\varepsilon)$.

Lemma 4. Let $g^{*}=\Gamma(g)$ with $g \in G(\varepsilon)$ any $\varepsilon>0$. Then $g^{*}: R^{J} \rightarrow R$ is continuous on $Y$.

Lemma 5. Let $g^{*}=\Gamma(g)$ with $g \in G(\varepsilon)$ any $\varepsilon>0$. Let $\Delta=\left(\Delta_{1}, \ldots, \Delta_{J}\right)$, $\Delta_{j}=\delta>0, \Delta_{i}=0$ all $i \neq j$, and $y, y+\Delta \in Y$. Then $g(y+\Delta)=g(y)$ implies $g^{*}\left(y+\Delta^{\prime}\right)>g^{*}(y)$ each $\Delta^{\prime} \in(0, \Delta)$.

Lemma 6. Suppose $g^{*}=\Gamma(g)$ and $g \in G(\varepsilon)$. Let $\Delta=\left(\Delta_{1}, \ldots, \Delta_{J}\right)$ with $\Delta_{j}=\delta>0$ and $\Delta_{i}=0$ all $i \neq j$. Suppose $g(y)=g^{*}(y)$. Let $y, y+\Delta \in Y$. Then there exists an $\varepsilon^{*}>0$ such that $\varepsilon=\varepsilon^{*}$ and $g(y+\Delta)-g(y)=\delta \cdot \varepsilon^{*}$ some $\delta>0$ imply $g^{*}\left(y+\Delta^{\prime}\right)-g^{*}(y)<\delta^{\prime} \cdot \varepsilon^{*}$ some $\Delta^{\prime} \in(0, \Delta)$ with $\Delta_{j}^{\prime}=\delta^{\prime}$.

Lemma 4 shows that each $g^{*}$ is continuous (with respect to the conventional Euclidean metric). Lemmas 5 and 6 will enable us to prove that the fixed points of $\Gamma$ and $\Psi$ are the same. From this point forward $\varepsilon^{*}$ will refer to the constant derived in Lemma 6.

We need one more lemma before we can turn our attention to $\Psi$.

Lemma 7. $\Gamma$ is continuous (with respect to $\|\cdot\|$ ) on $G\left(\varepsilon^{*}\right)$.

We define $\Psi$ as follows. Let $y_{L}$ be as in Lemma 2, and let $g^{*}=\Gamma(g)$ with $g \in G\left(\varepsilon^{*}\right)$. Suppose $y(\delta)=\left(y_{L}(1)+\delta, y_{L}(2), \ldots, y_{L}(J)\right)$. For $y=y(\bar{\delta}) \in Y$ any $\bar{\delta} \geqslant 0$ define

$$
\begin{aligned}
h(y) & =\operatorname{Max}_{y_{L} \leqslant y(\delta) \leqslant y}\left\{g^{*}(y(\delta)), k_{L}\right\}, \\
h^{*}(y) & =\operatorname{Min}_{0 \leqslant \delta \leqslant \delta}\left\{h(y(\delta))+\varepsilon^{*} \cdot(\bar{\delta}-\delta)\right\} .
\end{aligned}
$$

After selecting each $\bar{\delta}$ with $y \in Y$, choose any $y_{1} \in\left[y_{t}(1), y_{U}(1)\right]$. Let $y(\delta)=$ $\left(y_{1}, y_{L}(2)+\delta, y_{L}(3), \ldots, y_{L}(J)\right)$. For $y=y(\bar{\delta}) \in Y$ any $\bar{\delta} \geqslant 0$ define

$$
h(y)=\operatorname{Max}_{y_{L, 1} \leqslant y(\delta) \leqslant y}\left\{g^{*}(y(\delta)), h\left(y_{L, 1}\right)\right\},
$$

where $y_{L, 1}=\left(y_{1}, y_{L}(2), \ldots, y_{L}(J)\right)$. Define $h^{*}(y)$ using line (10). Repeat the step for each $y_{1} \in\left[y_{L}(1), y_{U}(1)\right]$. Proceeding coordinate by coordinate, we have $h: Y \rightarrow R$ and $h^{*}: Y \rightarrow R$. Let

$$
\begin{aligned}
& \psi(g)=h, \\
& \Psi(g)=h^{*} .
\end{aligned}
$$


Then

LEMMA 8. $\Psi\left(G\left(\varepsilon^{*}\right)\right) \subset G\left(\varepsilon^{*}\right)$.

We also have

Lemma 9. $\Psi$ is continuous (with respect to $\|\cdot\|)$ on $G\left(\varepsilon^{*}\right)$.

Since $\Psi$ is continuous, $\Psi\left(G\left(\varepsilon^{*}\right)\right) \subset G\left(\varepsilon^{*}\right)$, and $G\left(\varepsilon^{*}\right)$ is convex and compact, Schauder's fixed-point theorem (see Smart [20]) proves that there is at least one $g=\Psi(g) \in G\left(\varepsilon^{*}\right)$. The following proposition shows that $g=$ $\Gamma(g)$ as well.

Proposition I. $g=\Psi(g) \in G\left(\varepsilon^{*}\right)$ implies $g=\Gamma(g)$. Thus, there is at least one $g \in G\left(\varepsilon^{*}\right)$ such that $g=\Gamma(g)$.

If $g \in G\left(\varepsilon^{*}\right)$, then $g$ is continuous and nondecreasing. In fact, Lemma 5 shows that $g=\Gamma(g) \in G\left(\varepsilon^{*}\right)$ implies $g$ must be increasing (on $Y$ ).

If $g=\Gamma(g) \in G\left(\varepsilon^{*}\right)$ and if all families use $g$ to predict the next-period aggregate capital stock, their behavior will bear their expectations out. So, if for each Borel set $A \subset R$,

$$
v(k, A)=\operatorname{Pr}\{g(y(k, \tilde{z})) \in A\},
$$

then $v$ will define a transition function for the economy consistent with rational expectations on the part of all consumers. The number $v(k, A)$ gives the probability that if $k_{t}=k, k_{t+1}$ will be an element of the set $A$. The invariant measures for $v$ are the topic of Section III.

Notice that our equilibrium expectation functions $g=\Gamma(g) \in G\left(\varepsilon^{*}\right)$ contrast to solutions of Arrow-Debreu-Bewley (see $[1,3,5]$ ) general equilibrium models if our consumers have different utility functions or labor endowments. For, suppose consumers are homogeneous. Then with a complete set of futures markets (for labor, investment securities, and output) all young workers at time $t$ will save the same amount, buy even shares of output at time $t+1$ in all contingencies, agree to supply the same amount of labor in each contingency, and buy the same share of each investment security. Futures prices must adjust so that the capital stock, labor supply, and total sale of output will be the same at time $t+1$ regardless of the value $z_{t+1}$ takes. In this situation there will be an Arrow-Debreu-Bewley equilibrium corresponding to each of our equilibria.

If consumers are heterogeneous, on the other hand, the equilibria of the two types of model will differ. With a complete set of futures markets, a highly risk averse family might at time $t$ trade some of its next-period purchasing power contingent on the event of a favorable $z_{t+1}$ to a less 
cautious family for extra purchasing power contingent on the event of an unfavorable $z_{t+1}$. In our model that cannot happen. Thus our equilibria will typically differ from those of a traditional general equilibrium model unless all families are identical.

\section{Steady States}

If all families use $g=\Gamma(g) \in G\left(\varepsilon^{*}\right)$ to compute their expectations, $v$ (defined in line (14)) gives the actual transition function for the aggregate capital stock. We are now ready to prove that $v$ has a finite number of ergodic sets in $K$ and that associated with each such ergodic set $E_{i}$ there is a unique stationary distribution function $D_{i}$. Notice that if $K^{* *}=$ $K \cup\left(k_{U}, \infty\right)$, the proof of Lemma 1 shows $k_{t} \in K^{* *}$ implies $k_{t+s} \in K$ some finite $s$ with probability 1 . The proof of Lemma 2 shows that any $k^{\prime} \in\left(0, k_{L}\right)$ will work as a lower bound for $K$, with $\left(k^{\prime}, k_{L}\right)$ being a collection of transitory states. Thus, if

$$
K^{*}=(0, \infty),
$$

the ergodic sets for $v$ on $K,\left\{E_{1}, \ldots, E_{N}\right\}$, are the ergodic sets for $v$ on $K^{*}$.

Since $g \in G\left(\varepsilon^{*}\right), K$ is an invariant set for $v$. Since $g \in G\left(\varepsilon^{*}\right)$ is continuous, Grandmont and Hildenbrand [8] establish the existence of at least one invariant measure for $v$ on $K$, therefore. However, we will make the following argument to show that $v$ has only a finite number of ergodic sets in $K$ and that none decompose into two or more cyclic subsets.

We can easily establish that $v(k, \cdot)$ is a probability measure and $v(\cdot, A)$ is a measurable function.

Lemma 10. Let $g=\Gamma(g) \in G\left(\varepsilon^{*}\right)$, and let $v$ be as in line (14). Then $v(k, \cdot)$ is a probability measure for each $k \in K$, and for each Borel $A \subset K$ $v(\cdot, A)$ is a measurable function on $K$.

The paragraph surrounding Proposition I shows that $g=\Gamma(g) \in G\left(\varepsilon^{*}\right)$ implies $g$ must be increasing as well as continuous. We can use that fact to prove that $v$ has a finite number of ergodic sets in $K$.

Proposition II. Suppose $g=\Gamma(g) \in G\left(\varepsilon^{*}\right)$ and $v$ is as defined in line (14). Then there are a finite number of ergodic sets $E_{1}, \ldots, E_{N} \subset K$ for $v$. Provided no $E_{i}$ can be decomposed into two or more cyclic subsets, with each $E_{i}$ we can associate a unique stationary distribution function $D_{i}$.

The proof requires Lemma 10 and the theorems contained in Chapter 5 of Doob [6]. We will prove that no $E_{i}$ can be decomposed into two or more cyclic subsets in a moment. 


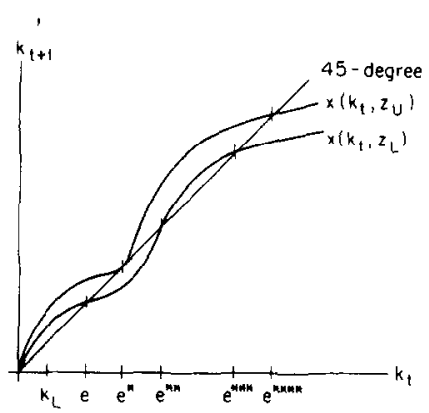

FIG. 1. The ergodic sets of $v$.

Define

$$
x(k, z)=g(y(k, z)) .
$$

Then $y$ is strictly increasing in both arguments, so the same is true for $x$. As Fig. 1 shows, Lemmas 1 and 2 imply that $x\left(k_{L}, z_{L}\right)>k_{L}$ and $x\left(k_{U}, z_{U}\right) \leqslant k_{U}$. Because of strict monotonicity, the $x$-curves for $z=z_{L}$ and $z_{U}$ do not touch at any $k_{t} \in K$. In the diagram there are two ergodic sets: $E_{1}=\left[e, e^{*}\right]$ and $E_{2}=\left[e^{* * *}, e^{* * * *}\right]$. Proposition II proves there must be at least one.

The points $k_{t} \in K-\left(E_{1} \cup E_{2}\right)$ represent transitory states. If $k_{t} \in\left[k_{L}, e\right]$, then $\lim _{s \rightarrow \infty} \operatorname{Pr}\left\{k_{t+s} \in E_{1}\right\}=1$. If $k_{t} \in\left[e^{* *}, e^{* * *}\right] \cup\left[e^{* * * *}, k_{l}\right]$, then $\lim _{s \rightarrow \infty} \operatorname{Pr}\left\{k_{t+s} \in E_{2}\right\}=1$. If $k_{t} \in\left[e^{*}, e^{* *}\right]$, then $k_{t+s} \in E_{1}$ or $E_{2}$ with probability 1 as $s \rightarrow \infty$. Thus, $k_{t} \in K$ (or $K^{*}$ ) implies the economy will end up in one ergodic set or the other with probability 1.

Once in an ergodic set such as $E_{1}, k$ will oscillate systematically among the cyclic subsets of $E_{1}$ if two or more such subsets exist. In the latter case there will be one stationary distribution $D_{1 a}$ for each cyclic set. If there are $n$ cyclic sets, $C_{1}, \ldots, C_{n} \subset E_{1}$, samplings at $n$-period intervals will fall in $C_{i}$ with frequencies determined by $D_{1 i}$. We rule out such a situation using assumption (A6).

Proposition III. Let $g=\Gamma(g) \in G\left(\varepsilon^{*}\right)$, and let $v$ be as in line (14). Let $E \subset K$ be ergodic. Then $E$ can have only one cyclic subset of positive measure.

Thus, if $k_{t} \in K^{*}$, we know that with probability $1 k_{t+s}$ will enter an ergodic set $E \in\left\{E_{1}, \ldots, E_{N}\right\}$ with $E_{i} \subset K$ all $i$ at some finite $s$. If $k_{t+s} \in E$, then $k_{t+s^{*}} \in E$ with probability 1 for each $s^{*}>s$. There will be a single distribution $D$ associated with $E$ such that $\int_{\mathrm{A}} D(d k)$ gives the frequency with which realizations $k_{t+s}, s \geqslant 0$ will fall in any Borel set $A \subset E$ over the very long run. ${ }^{7}$

\footnotetext{
'Let $v^{(1)}(k, A)=v(k, A)$ and $v^{(i+1)}(k, A)=\int_{k} v^{(i)}\left(k^{*}, A\right) v\left(k, d k^{*}\right)$ all $i \geqslant 1$. Then $\prod_{A} D(d k)=\lim _{i \rightarrow \infty} v^{(i)}(k, A)$ any Borel $A \subset E$ is a second way of looking at the role of $D$.
} 


\section{CONCLUSIONS}

We have proven the existence of an equilibrium transition function $v$ for our economy consistent with rational expectations on the part of all households. We also have shown that with each such $v$ we can associate $N$ ergodic sets $E_{i}$ (with $0<N<\infty$ ) and accompanying stable, unique stationary distribution functions $D_{i}$.

Although the random shocks in our model are intertemporally independent, Lemma 5 shows that for any equilibrium $g \in G\left(\varepsilon^{*}\right)$ we cannot have $g_{j+}(y)=0$ any $y \in Y$ and $j$, so non-serially correlated sequences of outcomes $\left\{k_{t}, k_{t+1}, \ldots\right\}$ are impossible. ${ }^{8}$ Thus, our model complements work done on "business-cycle models": models in the latter category generally study the role of shortsightedness or "nonrationality" (see, for example, Samuelson [19]) or incompletely revealed information (see, for example, Lucas [12] or [13]) in generating serially dependent outcome sequences from random demand shocks. Our shocks occur on the supply side of the model, but they generate serially dependent outcomes in spite of complete "rationality" in terms of behavior and expectations on the part of all market participants.

\section{APPENDIX}

1. Proof of Lemma 1. Assumption (A3) implies there exists $k_{U} \in(0, \infty)$ such that $\operatorname{Max}_{z \in Z}\left\{f\left(k_{U}, z\right)\right\}=k_{U}$. Let $k_{t} \in\left(0, k_{U}\right)$, and let $l=(1, \ldots, 1) \in R^{J}$. Then $k_{t+1} \leqslant l \cdot y\left(k_{t}, z_{t+1}\right) \leqslant f\left(k_{t}, z_{t+1}\right) \leqslant k_{U}$ any $z_{t+1} \in Z$.

2. Proof of Lemma 2. Let "lim" stand for " $\lim _{k_{L}, 0}$." Suppose (A4) applies for $j=1$. Let $g\left(y_{L}\right)=k_{L}$, and note that $y_{L}=y_{L}\left(k_{L}\right)$ depends on $k_{L}$. Let $s=y_{L}(1)-c_{1}$ if $c_{1}$ is the desired consumption now of young household $j=1$ given $y_{L}$ and $g$. Let $c_{2}(z)$ be the same household's anticipated consumption for next period if the next-period realization of $\tilde{z}$ is $z$.

Step 1. Let $c_{U}=w\left(k_{L}, z_{U}\right) \cdot l_{2}(1)+r\left(k_{L}, z_{U}\right) \cdot s$. Then $c_{2}(z) \leqslant c_{U}$ all $z \in Z$. (Al) and first-order conditions show $M R S\left(c_{1}, c_{l}\right) \equiv$ $u_{1}\left(c_{1}, c_{U}, 1\right) / u_{2}\left(c_{1}, c_{U}, 1\right) \geqslant E\left[u_{1}\left(c_{1}, c_{2}(\tilde{z}), 1\right)\right] / E\left[u_{2}\left(c_{1}, c_{2}(\tilde{z}), 1\right)\right] \geqslant r\left(k_{L}, z_{L}\right)$ any $k_{L} . \lim r\left(k_{L}, r_{L}\right)=\infty$. So, $\lim \operatorname{MRS}\left(c_{1}, c_{U}\right)=\infty$. Let $r=r\left(k_{L}, r_{L}\right), r^{*}=$ $r\left(k_{L}, z_{U}\right), w=w\left(k_{L}, z_{L}\right)$, and $w^{*}=w\left(k_{L}, z_{U}\right)$. Note that $y_{L}(1)=w \cdot l_{1}(1)$.

Suppose $\lim w\left(k_{L}, z\right)=0$ some $z \in Z$. Then (A4) implies the same holds for all $z \in Z$. $w \cdot l_{1}(1) \geqslant c_{1} \geqslant 0$, so $\lim w=0$ implies $\lim c_{1}=0$. Either $\lim c_{U}=0$ or $\lim r^{*} s \neq 0$. In the latter case $\lim r^{*} s / w=\infty$. If $\lim c_{U}=0, \lim$ $\operatorname{MRS}\left(c_{1}, c_{U}\right)=\infty$ and $\lim c_{1}=\lim c_{U}=0$. So, (A4) implies $\lim c_{U} / c_{1}=\infty$.

\footnotetext{
${ }^{8}$ Recall that $g_{i}^{+}(y)$ stands for the partial derivative from the right for $g$ at $y$.
} 
$c_{U} / c_{1}=\left[\left(w^{*} l_{2}(1) / w\right)+\left(r^{*} s / w\right)\right] /\left[l_{1}(1)-(s / w)\right]$. So, $\lim c_{U} / c_{1}=\infty$ implies $\lim r^{*} s / w=\infty$ or $\lim s / w=l_{1}(1)$. The latter implies $\lim r^{*} s / w=\infty$. Thus, $\lim w\left(k_{L}, z\right)=0$ all $z \in Z$ implies $\lim r^{*} s / w=\infty$.

Suppose $\lim w\left(k_{L}, z\right)>0$ all $z \in Z$. Suppose also that $\lim r^{*} s / w \neq \infty$. Then $\lim s / w=0=\lim s$. So, $\lim c_{1}=\lim w l_{1}(1)>0$. For some sequence $\left(k_{L}\right)_{n} \rightarrow 0$ we must have $r^{*} s \rightarrow B \in[0, \infty)$ as $\left(k_{L}\right)_{n} \rightarrow 0$. Let "lim*" stand for "limit as $\left(k_{L}\right)_{n} \rightarrow 0$," so that $\lim ^{*} r^{*} s=B$. Note that $\lim ^{*} w^{*} l_{2}(1)=\lim$ $w^{*} l_{2}(1)=A$ some $A \in[0, \infty)$. If $A+B>0, \lim ^{*} M R S\left(c_{1}, c_{U}\right)=M R S$ (lim* $\left.w l_{1}(1), A+B\right)<\infty$, a contradiction of $\lim M R S\left(c_{1}, c_{U}\right)=\lim *$ $\operatorname{MRS}\left(c_{1}, c_{U}\right)=\infty$ established above. If $A+B=0$, then $\lim ^{*} c_{1}>0, \lim ^{*}$ $c_{2}=0$, and $\lim ^{*} M R S\left(c_{1}, c_{U}\right)=\lim ^{*} r=\infty$. That contradicts (A1). So, lim $w\left(k_{L}, z\right)>0$ all $z \in Z$ implies $\lim r^{*} s / w=\infty$.

Step 2. $\infty=\lim r^{*} s / w=1 \cdot \lim \left(s / k_{L}\right) \cdot \lim \left(w^{*} / w\right) \cdot \lim \sigma\left(k_{L}, z_{U}\right)$. So, (A4) implies $\lim s / k_{L}=\infty$. Thus, $\lim \gamma\left(y_{L}\left(k_{L}\right), g\right) / k_{L} \geqslant \lim s / k_{L}=\infty$. So, there exists $k_{L} \in\left(0, k_{U}\right)$ with $\gamma\left(y_{L}\left(k_{L}\right), g\right)>k_{L}$ if $g\left(y_{L}\left(k_{L}\right)\right)=k_{L}$.

3. Proof of Lemma 3. Since $G(\varepsilon)$ is closed (see Munkres [16, p. 130]), it is compact by Ascoli's theorem (see [16, p. 277]) if it is equicontinuous. It is "equicontinuous" if for any $\eta>0$ and $y \in Y$ there exists $\delta>0$ such that $g \in G(\varepsilon), y^{\prime} \in Y$, and $\operatorname{Max}_{j}\left|y_{j}-y_{j}^{\prime}\right|<\delta$ implies $\left|g(y)-g\left(y^{\prime}\right)\right|<\eta$.

Fix any $\eta>0$. Suppose $y, y^{\prime} \in Y$. Define $y^{*}=\left(y_{1}^{*}, \ldots, y_{J}^{*}\right)$ with $y_{j}^{*}=$ $\operatorname{Min}\left\{y_{j}, y_{j}^{\prime}\right\}$. Define $y^{* *}$ with $y_{j}^{* *}=\operatorname{Max}\left\{y_{j}, y_{j}^{\prime}\right\}$. Let $g \in G(\varepsilon)$. Then $g\left(y^{*}\right) \leqslant$ $g(y), g\left(y^{\prime}\right) \leqslant g\left(y^{* *}\right)$.

We have $y^{*}, y^{* *} \in Y$. Suppose $\operatorname{Max}_{j}\left|y_{j}-y_{j}^{\prime}\right|<\delta \equiv \eta /(J \cdot \varepsilon)$. Then $\operatorname{Max}_{j}\left|y_{j}^{*}-y_{j}^{* *}\right|<\delta$. So, $\left|g(y)-g\left(y^{\prime}\right)\right| \leqslant g\left(y^{* *}\right)-g\left(y^{*}\right)<\delta \cdot J \cdot \varepsilon=\eta$. Thus $G(\varepsilon)$ is equicontinuous.

4. Proof of Lemma 4. Suppose $c_{1}(j)=c_{1}(j, y, g)$ maximizes $\xi\left(j, y, c_{1}, g\right) \equiv \int_{Z} u\left(c_{1}, \tilde{r} \cdot\left(y_{j}-c_{1}\right)+l_{2}(j) \cdot \tilde{w}, j\right) P(d z)$, where $\tilde{r}=r(g(y), \tilde{z})$, $w=w(g(y), z)$, and $y=\left(y_{1}, \ldots, y_{J}\right)$. Since $\xi$ is concave in $c_{1}$ and continuous in $y$, Berge's [2] "maximum theorem" shows $c_{1}(j, y, g)$ is continuous in $y$ each $j$. Thus $g^{*}(y)=\sum_{j=1}^{J}\left[y_{j}-c_{1}(j, y, g)\right]$ is continuous in $y$.

5. Proof of Lemma 5. $g(y+\Delta)=g(y)$ implies the partial derivative from the right $g_{j}^{+}\left(y^{*}\right)$ exists and equals 0 all $y^{*} \in[y, y+\Delta)$. First-order conditions show $c_{1}\left(i, y+\Delta^{\prime}, g\right)=c_{1}(i, y, g)$ all $\Delta^{\prime} \in(0, \Delta)$ and $i \neq j$.

Step 1. Let $u_{11}, u_{12}$, and $u_{22}$ be the partial derivatives of $u\left(c_{1}(j), c_{2}(j), j\right)$ if $c_{2}(j)=\tilde{r} \cdot\left(y_{j}^{*}-c_{1}(j)\right)+\tilde{w} \cdot l_{2}(j), \tilde{r}=r\left(g\left(y^{*}\right), \tilde{z}\right)$, and $\tilde{w}=w\left(g\left(y^{*}\right), \tilde{z}\right)$. Differentiating first-order conditions for $c_{1}(j), E\left[u_{11}-2 \cdot \tilde{r} \cdot u_{12}+\tilde{r}^{2} \cdot u_{22} \mid\right.$ $d c_{1}(j)=E\left[-\tilde{r} \cdot u_{12}+\tilde{r}^{2} \cdot u_{22}\right] d y_{j}$ at each $y^{*} \in[y, y+\Delta)$ if $d y_{j}>0$. Then (A1) shows $d c_{1}(j) / d y_{j}<1$. So, $g_{j}^{*+}\left(y^{*}\right)=1-d c_{1}(j) / d y_{j}>0$.

Step 2. Thus, $g_{j}^{*+}\left(y^{*}\right)>0$ each $y^{*} \in[y, y+\Delta)$. Since $g \in G(\varepsilon)$ and $u$ is twice continuously differentiable, $g_{j}^{*+}$ is continuous at each such $y^{*}$. So, 
$g^{*}\left(y+\Delta^{\prime}\right)-g^{*}(y) \geqslant \operatorname{Min}_{y^{*} \in\left[y, y+\Delta^{\prime}\right]} g_{j}^{*+}\left(y^{*}\right) \cdot \delta^{\prime}>0$ any $\Delta^{\prime} \in(0, \Delta)$ with $\Delta_{j}^{\prime}=\delta^{\prime}$.

6. Proof of Lemma 6. Suppose $g(y+\Delta)-g(y)=\delta \cdot \varepsilon$ and $g \in G(\varepsilon)$. Then $g_{j}^{+}\left(y^{*}\right)=\varepsilon$ all $y^{*} \in[y, y+\Delta)$. Let $y=\left(y_{1}, \ldots, y_{J}\right)$ be the vector of incomes for young families now, and let $k=g(y)$. Let $s_{m}=y_{m}-c_{1}(m)$ all $m$, and let $s=\sum_{m=1}^{J} s_{m}$. Then $s_{m} \geqslant 0$ all $m$ and $s=k$ if $g(y)=g^{*}(y)$. Let $g(y)=g^{*}(y)$.

Step 1. Let $\bar{r}=r\left(k_{L}, z_{U}\right)$. Assumption (A5) implies $r(k, z)+r_{1}(k, z)$. $k>0$ all $(k, z) \in K \times Z$. Thus there exists $r^{*}>\bar{r}$ such that $\left(r^{*} /\left(r^{*}+1\right)\right)$. $r(k, z)+r_{1}(k, z) \cdot k>0$ all $(k, z) \in K \times Z$. Fix $\varepsilon=1+r^{*}$.

Step 2. Set $d y_{j}=d x>0$ and $d y_{i}=0$ all $i \neq j$. Let $d s_{j}=d x+$ $\left(r^{*} \cdot s_{j} / s\right) d x$ and $d s_{i}=\left(r^{*} \cdot s_{i} / s\right) d x$ all $i \neq j$. Then $d s \equiv \sum_{m=1}^{J} d s_{m}=\varepsilon \cdot d x$ and $d s_{m} \geqslant 0$ all $m$.

Step 3. Let $r=r(g(y), z)$, where $z$ is the next-period realization of $\tilde{z}$. Then $d\left(r \cdot s_{m}\right) / d y_{j}=r\left(d s_{m} / d y_{j}\right)+s_{m} r_{1} g_{j}^{+}(y) \geqslant\left(r r^{*} s_{m} / s\right)+s_{m} r_{1} \varepsilon=\varepsilon$. $\left[\left(r^{*} / \varepsilon\right) \cdot r+r_{1} k\right] \cdot\left(s_{m} / s\right) \geqslant 0$ by Step 1 with strict inequality when $s_{m}>0$.

Step 4. Step 3, (A1), and the first-order conditions for $c_{1}(m)$ show that if $y$ changes to $y+\Delta^{\prime}$, where $\Delta^{\prime} \in(0, \Delta)$, and if each family's saving is changed by $\delta_{j}^{\prime}$, where $\delta_{j}^{\prime}=\delta^{\prime}+\left(r^{*} s_{j} / s\right) \delta^{\prime}$ or $\delta_{i}^{\prime}=\left(r^{*} s_{i} / s\right) \delta^{\prime}$ when $i \neq j$, then provided $\delta^{\prime}=\Delta_{j}^{\prime}$ is small enough, each family of type $m$ will want to decrease the change in its saving if $s_{m}>0$ or $m=j$ and leave it unchanged otherwise.

Suppose $\delta_{m}^{*}$ is the desired change in saving for family $m$ following the change from $y$ to $y+\Delta^{\prime}$ above. Suppose $\sum_{m=1}^{J} \delta_{m}^{*}>\sum_{m=1}^{J} \delta_{m}^{\prime}$. Let $k^{*}=k+$ $\sum_{m=1}^{J} \delta_{m}^{*}$ and $k^{\prime}=k+\sum_{m=1}^{J} \delta_{m}^{\prime}$. Then $k^{*}>k^{\prime}$ so (A5) implies $r\left(k^{*}, z\right)$. $k^{*}>r\left(k^{\prime}, z\right) \cdot k^{\prime}$ all $z \in Z$ (assuming $k^{*}, k^{\prime} \in K$ ). Thus for some $m$ we have $r\left(k^{*}, z\right) \cdot\left(s_{m}+\delta_{m}^{*}\right)>r\left(k^{\prime}, z\right) \cdot\left(s_{m}+\delta_{m}^{\prime}\right)$ any $z \in Z$. So, the arguments above show the first-order conditions of family $m$ are being violated at $s_{m}+\delta_{m}^{*}$, a contradiction. So, $\sum_{m=1}^{J} \delta_{m}^{*}>\sum_{m=1}^{J} \delta_{m}^{\prime}$ is not possible.

If $0<\sum_{m=1}^{J} \delta_{m}^{*} \leqslant \sum_{m=1}^{J} \delta_{m}^{\prime}$, the first paragraph of Step 4 shows $\delta_{m}^{*} \leqslant \delta_{m}^{\prime}$ all $m$, with strict inequality for at least one $m$. Thus, $g^{*}\left(y+\Delta^{\prime}\right)-g^{*}(y)=$ $\sum_{m=1}^{J} \delta_{m}^{*}<\sum_{m=1}^{J} \delta_{m}^{\prime}=\varepsilon \cdot \delta^{\prime}$.

7. Proof of Lemma 7. Suppose $g, \bar{g} \in G\left(\varepsilon^{*}\right)$ and $\|g-\bar{g}\|=\delta$. Let $g^{*}=$ $\Gamma(g)$ and $\bar{g}^{*}=\Gamma(\bar{g})$.

Maximizing over $(k, z) \in K \times Z$, let $r_{1}^{*}=\operatorname{Max}\left\{\left|r_{1}(k, z)\right|\right\}$ and $w_{1}^{*}=$ $\operatorname{Max}\left\{w_{1}(k, z)\right\}$. Let $s_{i}$ be the saving of young household $i$ at present given $g$, and let $\overline{s_{i}}$ be the same given $\bar{g}$. Then first-order conditions show $\left|s_{i}-\overline{s_{i}}\right| \leqslant \delta x$ for all $i$ where $x=k_{U} r_{1}^{*}+l w_{1}^{*}$. So, $\left\|g^{*}-\bar{g}^{*}\right\| \leqslant \delta \cdot x \cdot J$. Thus, $\Gamma$ is continuous. 
8. Proof of Lemma 8. Let $y, y^{\prime} \in Y$. Set $y_{i}^{*}=\operatorname{Min}\left\{y_{i}, y_{i}^{\prime}\right\}$ and $y_{i}^{* *}=$ $\operatorname{Max}\left\{y_{i}, y_{i}^{\prime}\right\}$ all $i$. Then $y^{*} \leqslant y, y^{\prime} \leqslant y^{* *}$ and $y, y^{*} \in Y$. We have $\mid h^{*}(y)-$ $h^{*}\left(y^{\prime}\right) \mid \leqslant h^{*}\left(y^{* *}\right)-h^{*}\left(y^{*}\right) \leqslant \sum_{i=1}^{J}\left[h^{*}\left(y^{* *}(i)\right)-h^{*}\left(y^{*}(i)\right)\right]$, where $y^{*}(1)=y^{*}, \quad y^{*}(2)=\left(y_{1}^{* *}, y_{2}^{*}, \ldots, y_{J}^{*}\right), \quad y^{*}(3)=\left(y_{1}^{* *}, \quad y_{2}^{* *}, \quad y_{3}^{*}, \ldots, y_{J}^{*}\right), \ldots$, $y^{* *}(1)=y^{*}(2), y^{* *}(2)=y^{*}(3), \ldots$, and $y^{* *}(J)=y^{* *}$. Lines $(9)-(11)$ and Lemma 4 show the terms in brackets are continuous.

9. Proof of Lemma 9. Let $g, \bar{g} \in G\left(\varepsilon^{*}\right)$. Let $h=\psi(g), h^{*}=\Psi(g)$, $\bar{h}=\psi(\bar{g}), \bar{h}^{*}=\Psi(\bar{g}), g^{*}=\Gamma(g)$, and $\bar{g}^{*}=\Gamma(\bar{g})$.

Step 1. Claim $\left\|g^{*}-\bar{g}^{*}\right\| \geqslant\|h-\bar{h}\|$. Choose any $y \in Y$. Suppose $h(y)>$ $\bar{h}(y)$. Then $h(y)=g^{*}(x)$ some $x \leqslant y, x \in Y$ or $h(y)=k_{L}$. In the latter case $h(y)=\bar{h}(y) . \quad$ Otherwise, $\quad|h(y)-\bar{h}(y)|-h(y)-\bar{h}(y) \leqslant g^{*}(x)-\bar{g}^{*}(x) \leqslant$ $\left\|g^{*}-\bar{g}^{*}\right\|$. Since this holds for any $y \in Y,\|h-\bar{h}\| \leqslant\left\|g^{*}-\bar{g}^{*}\right\|$.

Step 2. Claim $\left\|h^{*}-\bar{h}^{*}\right\| \leqslant\|h-\bar{h}\|$. Let $y \in Y$. Suppose $h^{*}(y)>\bar{h}^{*}(y)$. Then $\bar{h}^{*}(y)=\bar{h}(x)+\varepsilon^{*} \cdot \sum_{j=1}^{J}\left(y_{j}-x_{j}\right)$ some $x$ with $y_{L} \leqslant x \leqslant y$. So, $\mid h^{*}(y)$ $-\bar{h}^{*}(y) \mid=h^{*}(y)-\bar{h}^{*}(y) \leqslant h(x)+\varepsilon^{*} \cdot \sum_{j=1}^{J}\left(y_{j}-x_{j}\right)-\bar{h}(x)-\varepsilon^{*}$. $\sum_{j=1}^{J}\left(y_{j}-x_{j}\right)=h(x)-\bar{h}(x) \leqslant\|h-\bar{h}\|$.

Step 3. Let any $\eta>0$ be given. Lemma 7 implies there exists a $\delta>0$ such that $\left\|g^{*}-\bar{g}^{*}\right\|<\eta$ if $\|g-\bar{g}\|<\delta$. Combining this with steps 1 and 2 above, $\|g-\bar{g}\|<\delta$ implies $\left\|h^{*}-\bar{h}^{*}\right\|<\eta$. Thus $\Psi$ is continuous.

\section{Proof of Proposition I. Let $g=\Psi(g) \in G\left(\varepsilon^{*}\right)$. Let $g^{*}=\Gamma(g)$.}

Step 1. $g^{*}\left(y_{L}\right)=g\left(y_{L}\right)$ unless $g^{*}\left(y_{L}\right)<k_{L}=g\left(y_{L}\right)$. But, Lemma 2 rules out the latter. Hence, $g^{*}\left(y_{L}\right)=g\left(y_{L}\right)$, and in fact both exceed $k_{L}$.

Step 2. Let $x(\delta)=\left(y_{L}(1)+\delta, y_{L}(2), \ldots, y_{L}(J)\right) . \quad$ Let $\quad \bar{\delta}=\inf \{\delta>0 \mid$ $\left.g^{*}(x(\delta)) \neq g(x(\delta))\right\}$.

Suppose $g^{*}(x(\bar{\delta}+\eta))<g^{*}(x(\bar{\delta}))$ all $\eta \in(0, \bar{\eta})$ some $\bar{\eta}>0$. Then $g=\Psi(g)$ implies $g(x(\bar{\delta}+\eta))=g(x(\bar{\delta}))$ all such $\eta$. So, Lemma 5 implies $g^{*}(x(\bar{\delta}+\eta))>g^{*}(x(\bar{\delta}))$ some $\eta \in(0, \bar{\eta})$, a contradiction.

Suppose $g^{*}(x(\bar{\delta}+\eta))-g^{*}(x(\bar{\delta}))>\varepsilon^{*} \cdot \eta$ all $\eta \in(0, \bar{\eta})$ some $\bar{\eta}>0$. Then $g=\Psi(g)$ implies $g(x(\bar{\delta}+\eta))-g(x(\bar{\delta}))=\varepsilon^{*} \cdot \eta \quad$ all $\quad \eta \in(0, \bar{\eta})$. Then Lemma 6 implies $g^{*}(x(\bar{\delta}+\eta))-g^{*}(x(\bar{\delta}))<\varepsilon^{*} \cdot \eta$ some $\eta \in(0, \bar{\eta})$, a contradiction of our supposition.

So, $g(x(\delta))=g^{*}(x(\delta))$ all $\delta \geqslant 0$.

Step 3. Step 2 applies for $x(\delta)=\left(y_{1}, y_{L}(2)+\delta, y_{L}(3), \ldots, y_{L}(J)\right)$ any $y_{1} \in$ $\left[y_{L}(1), y_{U}(1)\right]$. And, continuing coordinate by coordinate, for $x(\delta)=$ $\left(y_{1}, \cdot, y_{i-1}, y_{L}(i)+\delta, y_{L}(i+1), \ldots, y_{L}(J)\right)$ any $i \leqslant J$ and any $\left(y_{1}, \ldots, y_{i-1}\right) \in$ $\prod_{j=1}^{i-1}\left[y_{L}(j), y_{U}(j)\right]$. Thus, $g(x)=g^{*}(x)$ all $x \in Y$.

11. Proof of Lemma 10. $v(k, \cdot)$ is a probability measure by definition.

Suppose $A \subset K$ is a closed interval. Lemma 5 shows $g=\Gamma(g)$ implies $g$ is 
increasing. So, $B(k)=\{z \in Z \mid g(y(k, z)) \in A\}$ is a bounded, closed interval. Because $g$ and $y$ are continuous, $B$ is upper semi-continuous. Thus, $v(k, A)=$ $\int_{B(k)} P(d z)$ is continuous in $k$ by $(\mathrm{A} 6)$. So, $v(\cdot, A)$ is measurable.

The class of closed intervals generates the class of Borel sets of $K$. Thus, for any Borel $A \subset K$, any $k \in K$, and any positive integer $n$, we can find disjoint, closed intervals $C_{1}, \ldots, C_{m(n)}$ with $A \subset \bigcup_{i=1}^{m(n)} C_{i}$ and $0 \leqslant$ $v\left(k, \bigcup_{i=1}^{m(n)} C_{i}\right)-v(k, A)<1 / n$. Yet, $\quad v\left(k, \bigcup_{i=1}^{m(n)} C_{i}\right)=\sum_{i=1}^{m(n)} v\left(k, C_{i}\right)$ is measurable by the preceding argument. So we have a sequence of measurable functions $v\left(k, \bigcup_{i=1}^{m(n)} C_{i}\right) \rightarrow v(k, A)$ pointwise for each $k \in K$. Thus, $v(\cdot, A)$ is measurable on $K$ (see Halmos [10, p. 84]).

12. Proof of Proposition II. Let $\mu$ be the Lebesgue measure. Suppose there exists a constant $\eta>0$ such that for any Borel $A \subset K$ with $\mu(A)<\eta$ we have $v(k, A)<1-\eta$ all $k \in K$. Then Chapter 5 of Doob [6] shows that given Lemma 10 we will have $N$ ergodic sets with $N<\mu(K) / \eta$. Doob's Theorem 5.7 shows that with each $E_{i}$ we can associate a unique stationary $D_{i}$ given our hypotheses.

We must show that $\eta>0$ exists.

Step 1. Let $x(k, z)=g(y(k, z))$ and $\phi\left(k, z^{\prime}, z\right)=x\left(k, z^{\prime}\right)-x(k, z)$. Then $\phi$ is continuous and $\phi\left(k, z^{\prime}, z\right)>0$ if $k \in K ; z, z^{\prime} \in Z$; and, $z^{\prime}>z$. Let $\phi^{*}=$ $\operatorname{Min}_{k \in K}\left\{\phi\left(k, z_{U}, z_{L}\right)\right\}$. Then $\phi^{*}>0$.

Step 2. Note that $\mu(K) \geqslant \phi^{*}$. Let $\zeta=\phi^{*} / 2$. Suppose $A \subset K$ and $\mu(A)<\zeta$. Let $B(k)=\{z \in Z \mid x(k, z) \in A\}$ and $C(k)=Z-B(k)$. Let $\psi(k, z, \delta)=$ $\phi(k, z+\delta, z) / \delta$. Let $W^{*}=\operatorname{Max}_{(k, z) \in K \times z}\left\{l \cdot w_{2}(k, z)\right\}$. Then $\psi(k, z, \delta) \leqslant \varepsilon^{*} W^{*}$ all $k \in K ; z, z+\delta \in Z ;$ and, $\delta>0$. So, $\mu(C(k)) \cdot \varepsilon^{*} \cdot W^{*} \geqslant \mu(K-A)=$ $\mu(K)-\mu(A)>\phi^{*}-\zeta=\zeta$. Thus, $\mu(C(k))>\zeta /\left(\varepsilon^{*} W^{*}\right)$.

Assumption (A6) implies $p(z) \geqslant p_{L}$ all $z \in Z$ some $p_{L}>0$. Thus, $v(k, A)=$ $\int_{B(k)} p(z) d z=\int_{Z} p(z) d z-\int_{C(k)} p(z) d z<1-p_{L} \zeta /\left(\varepsilon^{*} W^{*}\right)$ any $k \in K$.

Step 3. Let $\eta=\operatorname{Min}\left\{\zeta, p_{L} \zeta /\left(\varepsilon^{*} W^{*}\right)\right\}$. Then $\mu(A)<\eta$ and $A \subset K$ imply $v(k, A)<1-\eta$ any $k \in K$.

13. Proof of Proposition III. Suppose $C$ is a cyclic subset of $E$ and $\mu$ is the Lebesgue measure. Let $k \in E$ with $v(k, C)=1$. Figure 1 shows $x(k, z)=$ $g(y(k, z))$ implies $x\left(k, z_{U}\right) \geqslant k \geqslant x\left(k, z_{L}\right)$. Then $I=\left(x\left(k, z_{L}\right), x\left(k, z_{r}\right)\right)$ implies $\mu(I)>0$ (by Lemma 5 ) and $\mu(I \cap C)=\mu(I)$.

Let $k^{*} \in I \cap C . x\left(k^{*}, z_{U}\right) \geqslant k^{*} \geqslant x\left(k^{*}, z_{L}\right)$. Let $B\left(k^{*}\right)=\left\{z \in Z \mid x\left(k^{*}, z\right) \in\right.$ $I \cap C\}$. Then $\mu\left(B\left(k^{*}\right)\right)>0$. So, $v\left(k^{*}, C\right) \geqslant v\left(k^{*}, I \cap C\right)=\int_{B\left(k^{*}\right)} p(z) d z>0$ by (A6). Since this result holds for any $k^{*} \in I \cap C$, and since $\mu(I \cap C)>0$, there cannot be a second cyclic subset of positive measure. ${ }^{9}$

\footnotetext{
${ }^{9}$ In other words, we have shown that the period of $C$ is 1 .
} 


\section{ACKNOWLEDGMENT}

I wish to thank my colleague Larry Blume for helpful comments at an early stage in this research.

\section{REFERENCES}

1. K. J. ARrow and F. H. Hahn, "General Competitive Analysis," Holden-Day, San Francisco, 1971.

2. C. Berge, "Topological Spaces," trans. E.M. Patterson, Oliver \& Boyd, Edinburgh, 1963.

3. T. F. BEWLEY, Existence of equilibria in economies with infinitely many commodities, $J$. Econ. Theory 4 (1972), 514-540.

4. W. Brock and L. Mirman, Optimal economic growth and uncertainty: the discounted case, J. Econ. Theory 4 (1972), 479-513.

5. G. Debreu, "Theory of Value," Yale Univ. Press, New Haven, 1973.

6. J. DooB, "Stochastic Processes," Wiley, New York, 1953.

7. J.M. Grandmont, Temporary general equilibrium theory, Econometrica 45 (1977), $535-572$.

8. J.M. Grandmont and W. Hildebrand, Stochastic processes of temporary equilibria, $J$. Math. Econ. 1 (1974), 247-278.

9. J. R. Green And M. Majumdar, The nature of stochastic equilibria, Econometrica 43 (1975), 647-660.

10. P. R. Halmos, “Measure Theory," Springer-Verlag, New York, 1974.

11. F. S. INABA, Consumption loans under uncertainty, J. Econ. Theory 15 (1977), 26-53.

12. R. E. LuCAS JR., Expectations and the neutrality of money, J. Econ. Theory 4 (1972), $103-124$.

13. R. E. Lucas JR., An equilibrium model of the business cycle, J. Pol. Econ. 83 (1975), 1113-1144.

14. L. J.MIRMAN, On the existence of steady state measures for one sector growth models with uncertain technology, Internat. Econ. Rev. 13 (1972), 271-286.

15. L. J. MiRman, The Steady State behavior of a class of one sector growth models with uncertain technology, J. Econ. Theory 6 (1973), 219-242.

16. J. R. Munkres, "Topology," Prentice-Hall, Englewood Cliffs, N. J., 1975.

17. R. RADNER, Existence of equilibrium of plans, prices, and price expectations in a sequence of markets, Econometrica 40 (1972), 289-304.

18. R. RADNER, Market equilibrium and uncertainty: concepts and problems, in "Frontiers of Quantitative Economics, Vol. II" (M. D. Intriligator and D. A. Kendrick, Eds.), North-Holland, Amsterdam, 1974.

19. P. A. Samuelson, Interactions between the multiplier analysis and the principle of acceleration, Rev. Econ. Statist. 21 (1939), 75-78.

20. D. R. Smart, "Fixed Point Theorems," Cambridge Univ. Press, London, 1974. 